\title{
Research of Performance of Static and Dynamic Routing Protocol Based on NS2
}

\author{
Hui Zheng', Siping Hu*, ${ }^{2}$, Shejie $\mathrm{Lu}^{2}$, Wuxiong $\mathrm{Xu}^{2}$ \\ ${ }^{1}$ Nanyang Institute of Technology, Nanyang, 473000, China \\ ${ }^{2}$ Hubei University of Science and Technology, Xianning, 430070, China \\ *E-mail: zjlg0001@126.com
}

Keywords: vehicle-mounted Ad Hoc network; routing protocol; packet loss rate; throughput;

\begin{abstract}
This paper mainly introduces the wireless mobile Ad Hoc network used for vehicle-to-vehicle communication. It also introduces the concepts of Ad Hoc network, vehicle-mounted Ad Hoc network and typical routing protocols. In this paper, 11 mobile nodes in the vehicle-based Ad Hoc network are studied, and the typical AODV and DSDV routing protocols are simulated in the LINUX environment using the famous simulation software NS2. Through the analysis of the simulation result data, the performance indexes of the two routing protocols are analyzed using performance indicators such as route throughput, jitter, packet loss rate, and end-to-end delay. The final result is that AODV performance is better than DSDV.
\end{abstract}

\section{Introduction}

Mobile Ad Hoc Network (Ad Hoc Network or MANET) is a multi-hop temporary ad hoc system composed of mobile nodes with wireless transceivers. It is a kind of self-organizing system that does not depend on fixed infrastructures. Wireless mobile network, as shown in Figure 1. The predecessor of the Ad Hoc network is a packet radio network, which allows the message exchange technology to operate in an environment that is not limited by a fixed or limited infrastructure. Figure 2-1 Each terminal node in the network has a routing function. It is responsible for discovering and maintaining routes to other nodes and transmitting or forwarding packets to neighboring nodes. Therefore, an Ad Hoc network is usually a multi-hop network and can pass through. The wireless connection constitutes an arbitrary network topology. This network can work independently or it can be connected to the Internet or a cellular wireless network. In addition, nodes can move freely and enter and exit the network autonomously, making the topology of the network often subject to rapid and unpredictable changes. Such a network can operate as a stand-alone network and can also be connected to a wired backbone network (such as the Internet). Compared with traditional cellular systems, Ad Hoc networks have the greatest advantage of not relying on base stations, strong self-organization and high mobility.

\section{Application of 2 Ad Hoc Networks}

The on-vehicle Ad Hoc network is an Ad Hoc network formed by self-organization of vehicles equipped with communication facilities without using any pre-architecture infrastructure. The vehicle-mounted ad hoc network is an ad hoc network designed specifically for inter-vehicle communication. The basic idea of the vehicle-mounted Ad Hoc network is that vehicles within a certain communication range can exchange their own speed, location, and other information and data sensed by the onboard sensors. Forward to drivers who are helpful to their passengers, improve driving safety, comfort and road usage, meet passengers' needs for various information, and automatically establish a mobile network. In the vehicle-mounted Ad Hoc network, cars will no longer be isolated units, but will become active network nodes. Node (vehicle) single-hop communication range is only a few hundred meters to a kilometer, each node (vehicle) is only a transceiver, but it is also a router, so the use of multi-hop data forwarding to more distant vehicle.

This technology creatively applies the ad hoc network to vehicle-to-vehicle communication, 
enabling the driver to obtain status information (such as vehicle speed, direction, position, brake pedal pressure, etc.) and real-time road condition information of other vehicles within the over-the-horizon range. The design goal of the onboard ad hoc network is to establish a platform for inter-vehicle communication, which not only improves traffic efficiency, but also brings reliable security and multiple conveniences to the driver's traffic, making the traveler more comfortable. It can be said that VANET is one of the most promising applications.

In addition to being able to implement local communication via a separate network, the on-board Ad Hoc network can also be connected to other fixed or mobile communication networks through fixed facilities (street lights, gas stations, etc.) as gateways for access points, providing more plentiful information. The function shows how flexible its networking is.

Since the 1990s, research on mobile Ad Hoc networks has been on the rise in the world and has gradually expanded from a small branch in the field of wireless communications to a relatively independent area. At present, there are more and more academic conferences for periodic Ad Hoc networks, both internationally and regionally (Europe and Asia, etc.). Ad Hoc network provides a wide range of application prospects. In addition to the military field, it is also widely used in emergency services, disaster recovery, environmental monitoring, personal networks, home networks, search and rescue, sensor networks and other fields, taking into account the Ad Hoc network Advantages: Does not rely on base stations, strong self-organization, high mobility, no center and self-organization, and dynamically changing network topology, it is gradually being applied to an emerging field - vehicle-mounted communications, and has therefore been academia And the industry's wide attention.

\section{Destination Sequence Distance Vector Routing Protocol (DSDV)}

The DSDV (Destination-Sequenced Distance-Vector) protocol is a pre-emptive routing protocol, which is improved from the traditional Bellman-Ford routing protocol. It features the use of the destination node sequence number to solve the routing loop and infinite count problem of the DBF algorithm. . DSDV is based on routing information protocol. It is only applicable to bidirectional links and is an early development of Ad Hoc routing protocol.

When DSDV is used, each mobile node in the network needs to maintain a routing table. The routing table entry includes the destination node, the number of hops, and a sequence number noted by the destination node. The serial number can help the node to distinguish valid and expired routes. Information and prevent routing loops. Routing information marked with a larger sequence number is always received. If two update packets have the same sequence number, the one with the smallest number of hops is selected and the route is optimized. Each node must periodically exchange routing information with neighboring nodes. Of course, routing updates can be triggered based on changes in the routing table. There are two ways to update the routing table: one is to update all, that is, the topology update message will include the entire routing table, which is mainly used in the case of rapid network change; the other way is incremental update, and the update message only contains changes. The routing part of the network is usually applied when the network changes slowly.

\section{Performance Analysis}

Two typical routing algorithms are simulated by NS network simulator. The mesh topology of Figure 1 below has 11 nodes in the set scene. The distance between each two adjacent nodes is $250 \mathrm{~m}$, forming a straight line. In the simulation time of $200 \mathrm{~s}$, the link flow is examined, and their average throughput, average delay, jitter, and average packet loss ratio under the AODV and DSDV protocols are compared. 


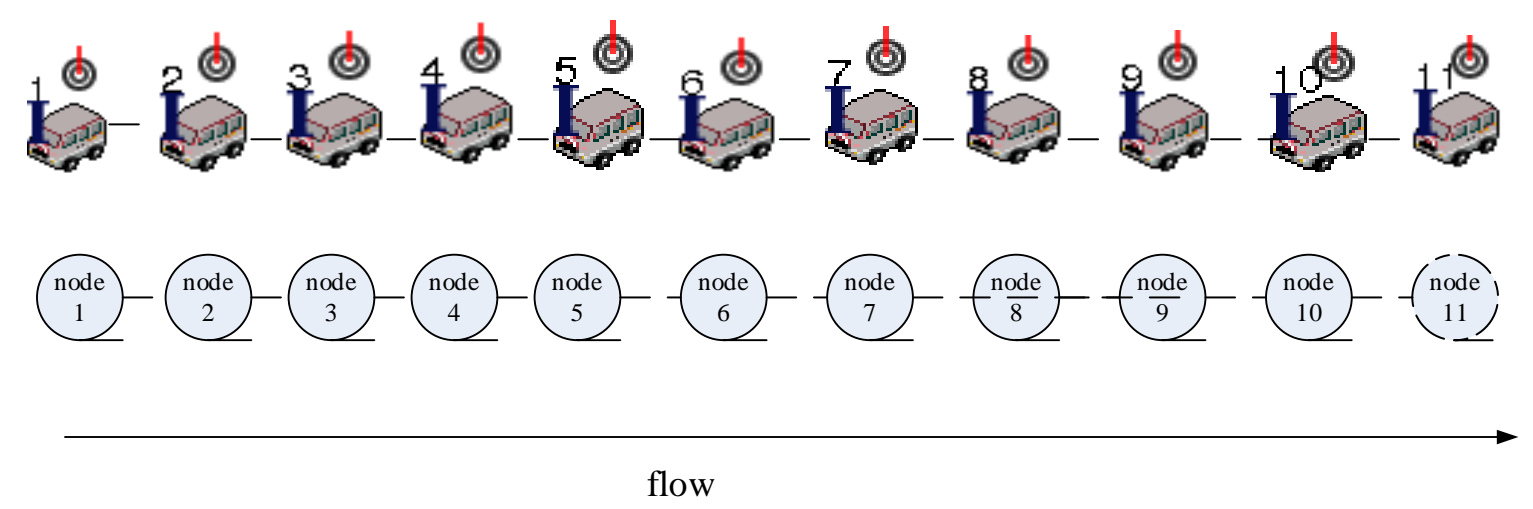

Figure. 1 Network topology

From Figure 2, we can see that we found that AODV is smaller than DSDV at any hop count. When there is only one hop in the network, there is no packet loss in DSDV or AODV. When there are three hops in the network, the DSDV protocol has started packet loss. The protocol AODV still has no packet loss problem. However, as the number of hops increases, the packet loss rate of both increases significantly. When the hop counts from 3 to 4 hops, the AODV packet loss increases sharply. When the hop count increases from 4 to 8 hops, the rate of increase is similar. However, from 8 to 10 jumps, the AODV curve is relatively flat. Overall, AODV outperforms DSDV in packet loss.



Figure. 2 Average packet loss rate 


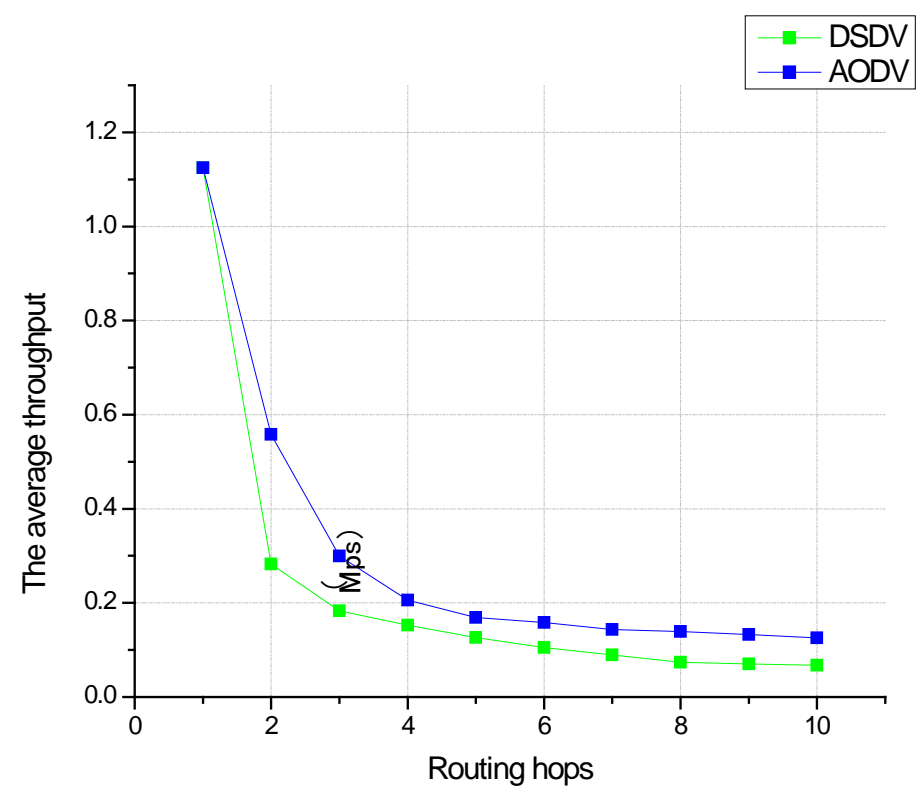

Figure 3 Average throughput

As shown in Figure 3, in the simulation time of 200s, as the number of nodes increases, that is, as the number of hops increases, the average throughput of protocols AODV and DSDV attenuates, and the attenuation rates of the two curves are similar. From a point of view, the throughput of the DSDV is smaller than that of the AODV at any jump. After the four jumps, both throughput curves tend to be stable and gradually approach zero. The size of the throughput can be used to evaluate the merits of a network performance. It is not difficult to find that the network performance of AODV is better than that of DSDV.

\section{Conclusion}

Two typical routing algorithms were simulated and compared by NS network simulator. When designing a network topology, it is often unrealistic to design a versatile routing protocol. A better solution is to use hybrid routing combined with a priori and reactive routing protocols, which can reduce the routing protocol overhead while minimizing packet delay. At present, a hierarchical routing algorithm based on clustering structure is proposed. In the cluster, a priori routing algorithm is used, and the clusters search for routes on demand. The cluster size can be adjusted adaptively according to the dynamic changes of the network. This routing algorithm draws on the advantages of two kinds of routing algorithms, with high efficiency and strong adaptability.

\section{Acknowledgements}

This research was supported by the Scientific Research Project of Education Department of Hubei Province under Grant B2018179, Doctor Initial Funding of Hubei University of Science and Technology (No. 2016-19XB003), the National Natural Science Foundation of China (No. 51479155).

\section{References}

[1] Ke C.H., Wei C.C., Lin K.W. A dynamic and adaptive transmission scheme for both solving uplink/downlink unfairness and performance anomaly problems in a multi-rate WLAN. Applied Mathematics \& Information Sciences, 2016, 6(4): 531S-537S.

[2] Yuan Z., Muntean G. iVoIP: an intelligent bandwidth management scheme for VoIP in WLANs. 
Journal of Wireless Networks, 2014, 20(3):457-473.

[3] Zawar S., Ather S., Imdad U. Effect of transmission opportunity and frame aggregation on VoIP capacity over IEEE 802.11n WLANs. 8th International Conference on Signal Processing and Communication Systems, 2014:1-7.

[4] Pilosof S., Ramachandran R., Raz D., et al. Understanding TCP fairness overwireless LAN, Proceedings of the IEEE INFOCOM, San Francisco, 2003: 863-872.

[5] Gong M.W., Wu Q., Williamson C. Queue management strategies to improve TCP fairness in IEEE 802.11 wireless LANs, Proceedings of the 4th International Symposium on Modeling and Optimization in Mobile, AdHoc, and Wireless Networks, Boston, 2006: 1-8.

[6] Kim S.W., Kim B.S., Fang Y. Downlink and uplink resource allocation in IEEE 802.11 wireless LANs, IEEE Transactions on Vehicular Technology, 2005, 1(54): 320-327.

[7] Cheng Y.H., Li Z.S., Zhu L. A bandwidth allocation scheme between uplink and downlink flows in IEEE 802.11 WLAN, Journal of South China University (Natural Science Edition), 2009, 5(37): $17-22$. 